\title{
Avaliação e análise da eficiência energética na irrigação em sistemas pivô central ${ }^{1}$
}

\author{
Aureo C. de Lima ${ }^{2}$, Sebastião C. G uimarães Jr. ${ }^{3}$, Carlos R. Fietz ${ }^{4}$ \& José R. Camacho ${ }^{3}$
}

\begin{abstract}
RESU MO
Com uma área irrigada aproximada de 710.500 ha no Brasil, é possível estimar um consumo anual de $1928 \mathrm{GWh}$ nos sistemas de irrigação por pivô central instalados. Considerando-se a necessidade de otimização da produção agrícola irrigada são analisadas, neste trabalho, as energias dissipadas nas tubulações de sucção, adutora, linha lateral, aspersão e proposta uma equação para determinar a potência útil de um sistema de irrigação por pivô central. Três sistemas pivô central do Assentamento Itamarati I, instalados em Ponta Porã, MS, foram avaliados e medidas de eficiência energética foram simuladas visando determinar uma configuração economicamente viável ao longo da vida útil do equipamento. $0 \mathrm{~s}$ sistemas foram caracterizados quanto ao aproveitamento da energia e a eficiência energética na irrigação média aumentou de 14,9 para 25,9\%, possibilitando a redução de $\mathrm{R} \$ 118.800$ por ano em 7.200 ha, 0 equival ente a $11 \%$ das despesas anuais com energia elétrica de toda a propriedade.
\end{abstract}

Palavras-chave: potência dissipada, ensaio, consumo específico, avaliação econômica, caracterização energética

\section{Evaluation and analysis of the energetic efficiency of center pivot irrigation systems}

\begin{abstract}
With an irrigated area of approximately 710,500 ha in Brazil, it is possible to estimate an annual electricity consumption of $1,928 \mathrm{GW}$ h per year due to center pivot systems. Considering the need of optimization of the irrigated agricultural production, the energies that are dissipated in the suction pipes, water supply, lateral lines and sprinkler systems are analyzed in this study and an equation is proposed to determine the useful power of center pivot irrigation systems. Three center pivot systems located in Itamarati I (a Government settlement for the landless in the city of Ponta Porã, State of Mato Grosso do Sul, Brazil) were evaluated and measures of energetic efficiency were simulated in order to determine a viable economic configuration to meet the life span of the equipment. The systems were characterized by the energy use and results show that the average irrigation energetic efficiency may be raised from 14.9 to $25.9 \%$ leading to a possible reduction of $\mathrm{R} \$ 118,800$ per year over 7,200 ha, equivalent to $11 \%$ of the yearly expenses with electricity of the whole area.
\end{abstract}

Key words: dissipated energy, computational simulation, specific consumption, economic evaluation, energetic characterization

\footnotetext{
1 Parte da tese de doutorado do primeiro autor

2 U FGD-Unidade II, Rodovia Dourados Itahum, km 12, C.P. 533, CEP 79801-970. Dourados, MS. Fone (67) 3411 - 3894. E-mail: aureolima@ufgd.edu.br

${ }^{3}$ N úcleo de Energia Rural e Fontes Alternativas de Energia/UFU, Av. Engenheiro Deniz 1178, CP 593, CEP 38400-902, U berlândia, M G. Fone: (34) 3239-4734. E-mail: scguimaes@ufu.br; jrcamacho@ufu.br

${ }^{4}$ Embrapa Agropecuária Oeste, BR 163, km 253, CP 661, CEP 79804-970, Dourados, MS. Fone: (67) 3416-9752. E-mail: fietz@cpao.embrapa.br
} 


\section{INTRODUÇÃO}

Normalmente os usuários de sistemas de irrigação não têm informações sobre o rendimento energético do equipamento e, de forma geral, adquirem projetos desenvolvidos pelos próprios fornecedores que, objetivando reduzir os custos iniciais, aumentam a relação potencia instalada por unidade de área do sistema de irrigação.

De acordo com Batista \& Coelho (2003), todo o sistema de bombeamento deve ser projetado levando em conta critérios econômicos, uma vez que o diâmetro da tubulação, a potência do sistema de bombeamento e as despesas operacionais, estão inter-relacionados.

A redução no diâmetro da tubulação eleva as perdas de carga e aumenta a potência necessária do conjunto motobomba; esta configuração de projeto propicia maior custo do conjunto elevatório e maior despesa com energia, embora proporcione economia na compra da tubulação. Por outro lado, aumentando-se o diâmetro da tubulação a potência do conjunto elevatório e o custo operacional de energia elétrica serão menores, podendo tornar-se economicamente viável ao longo da vida útil dos equipamentos.

A literatura apresenta vários métodos para determinação do diâmetro da tubulação de uma estação elevatória (Gomes, 2004); segundo este autor, a metodologia mais adequada constitui-se na introdução do critério econômico, de forma a se determinar a alternativa de projeto que minimize o custo de implantação e de operação, ao longo da vida útil do sistema.

Zocoler (2003) apresenta um estudo prático para determinação da melhor alternativa econômica, considerando os custos de implantação e operação ao longo da vida útil de um sistema pivô central. Gomes (2004), por sua vez, propõe a utilização do método baseado na variação linear dos custos das tubulações e o método das tentativas, enquanto CLASP (2005) apresenta um guia para etiquetagem e padronização da eficiência energética de equipamentos. Bernardo et al. (2005) indicam o dimensionamento da tubulação com base no limite das velocidades do fluido e no método de análise econômica.

Neste trabalho se propõe a extrapolação da análise econômica da adutora para os demais elementos de um pivô central, em que, a partir da redução da potência dissipada no motor, bomba, adutora, linha lateral e aspersores, se determinam as características de um projeto eficiente.

Esta análise é aplicada a três sistemas de irrigação por pivô central do Assentamento Itamarati I instalados em Ponta Porã, MS; a partir da avaliação inicial, se propõe alternativas para o aumento da eficiência energética e se caracterizam os equipamentos quanto ao aproveitamento da energia elétrica.

\section{MATERIAL E MÉTODOS}

A avaliação da eficiência energética proposta neste trabalho foi aplicada em três equipamentos instalados no Assentamento Itamarati I ensaiado em abril de 2006, localizado

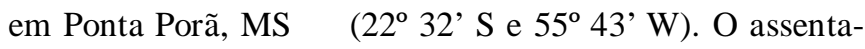
mento ocupa uma área de 25,1 mil ha na qual 1145 famílias irrigam 7,2 mil ha com 58 sistemas pivô central.

Os equipamentos de irrigação instalados há mais de 15 anos, totalizam 943 motores, somando a potência de 16.260 cv que, com consumo de 8,3 GWh de energia apresentaram, em 2004, um faturamento total de $\mathrm{R} \$ 1,08$ milhão. As principais características dos equipamentos avaliados estão apresentadas na Tabela 1.

\section{Potência dissipada no pivô}

A avaliação do conjunto motobomba foi realizada conforme ABNT (1975). Para cada pivô central foram medidas a potência de entrada no motor, a vazão e a pressão na saída da bomba. A perda de carga na sucção foi determinada pela equação de Darcy-Weisbach, Gomes (2004) e a altura manométrica na bomba pela equação de Bernoulli, (Batista \& Coelho, 2003). Obteve-se a potência do motor por meio do medidor universal de grandezas MUG - Elo Sistemas Eletrônicos; a vazão foi determinada através do medidor ultrasônico de vazão e velocidade de líquidos Fuji Portable Flowmeter "Portaflow X" e a pressão por um manômetro de 2 MPa instalado na saída da bomba. As espessuras das paredes das tubulações foram definidas pelo medidor ultra-sônico TI-10 Ultrasonic Thickness meter.

Determinaram-se as potências elétricas requeridas para dissipação da energia hidráulica ao longo da sucção, adutora e linha lateral dos sistemas, com base nas perdas de carga nas tubulações e no rendimento do conjunto motobomba (Eq. 1).

$$
\mathrm{P}_{\mathrm{Ed}}=\frac{\gamma \cdot \mathrm{Q}_{\mathrm{S}} \cdot \Delta \mathrm{h}}{\eta_{\mathrm{mb}}}
$$

em que:

$\mathrm{P}_{\mathrm{Ed}}$ - potência elétrica dissipada no elemento, $\mathrm{kW}$

$\gamma$ - peso específico da água a $4{ }^{\circ} \mathrm{C}, 9,806 \mathrm{kN} \mathrm{m}^{-3}$

$\Delta \mathrm{h}$ - perda de carga no elemento, mca

$\mathrm{Q}_{\mathrm{S}}$ - vazão bombeada pelo sistema hidráulico, $\mathrm{m}^{3} \mathrm{~s}^{-1}$

$\eta_{\mathrm{mb}}$ - rendimento do conjunto motobomba, adimensional

Determinaram-se, através de Sistema de posicionamento global (GPS) Garmin etrex modelo Vista, as coordenadas na captação, na bomba e no centro do pivô para obtenção dos comprimentos e desníveis das adutoras e se obtiveram a al-

Tabela 1. Características dos pivôs avaliados no Assentamento Itamarati, em abril de 2006

\begin{tabular}{|c|c|c|c|c|c|c|c|}
\hline & \multirow{2}{*}{ Pivô } & \multirow{2}{*}{$\begin{array}{l}\text { Potência do } \\
\text { motor (cv) }\end{array}$} & \multirow{2}{*}{$\begin{array}{l}\text { Área irrigada } \\
\text { (ha) }\end{array}$} & \multicolumn{2}{|c|}{ Adutora } & \multicolumn{2}{|l|}{ Linha lateral } \\
\hline & & & & diâmetro $(\mathrm{mm})$ & comprimento (m) & Comprimento $(\mathrm{m}) \mathrm{x}$ diâmetro $(\mathrm{mm})$ & comprimento \\
\hline 1 & L06 & 250 & 118,1 & 300 & 1142 & $234,5 \times 203+369,5 \times 168+9 \times 102$ & 613 \\
\hline 2 & $\mathrm{C} 08$ & 300 & 114,6 & 200 & 1466 & $592 \times 168+12 \times 102$ & 604 \\
\hline 3 & G13 & 300 & 108,0 & 300 & 2298 & $586,28 \times 168$ & 586 \\
\hline
\end{tabular}


timetria e o comprimento da linha lateral utilizando-se um nível Heerbrugg Wild NA 2 GPM3 e uma trena de $50 \mathrm{~m}$.

A eficiência de aplicação foi determinada segundo Oliveira et al. (2004) para $80 \%$ da área adequadamente irrigada e o Coeficiente de Heerman e Hein, $\mathrm{C}_{\mathrm{uH}}$, conforme ABNT (1998), com os coletores espaçados a $3 \mathrm{~m}$ em duas linhas com um ângulo de $3^{\circ}$.

Foram determinadas, durante a avaliação do pivô, a velocidade do vento, através de um Anemômetro ANM-10 da Hidrologia SA, a temperatura e a umidade do ar, com um termo-higrômetro MF972B da More Fitness.

Considerando a potência requerida para o bombeamento da água não disponibilizada à cultura, caracterizada pela Eficiência de Aplicação, Eq. 2, e a potência requerida para o funcionamento do emissor, Eq. 3, propõem-se, neste trabalho, a soma desas parcelas e a potência elétrica associada à aspersão, Eq. 4.

$$
\begin{gathered}
\mathrm{P}_{\mathrm{EdEA}}=\frac{\gamma \cdot \mathrm{H}_{\mathrm{m}} \cdot \mathrm{Q}_{\mathrm{S}}}{\eta_{\mathrm{mb}}} \cdot\left(1-\frac{\mathrm{EA}_{80}}{100}\right) \\
\mathrm{P}_{\mathrm{EdPA}}=\frac{\gamma \cdot \mathrm{Q}_{\mathrm{S}} \cdot \mathrm{EA}_{80} \cdot \mathrm{h}_{\mathrm{ASP}}}{100 \cdot \eta_{\mathrm{mb}}} \\
\mathrm{P}_{\mathrm{Edasp}}=\frac{\gamma \cdot \mathrm{Q}_{\mathrm{S}} \cdot \mathrm{EA}_{80}}{100 \cdot \eta_{\mathrm{mb}}} \cdot\left(\mathrm{H}_{\mathrm{m}} \cdot\left(\frac{100}{\mathrm{EA}_{80}}-1\right)+\mathrm{h}_{\mathrm{ASP}}\right)
\end{gathered}
$$

em que:

$\mathrm{P}_{\mathrm{EdEA}}$ - potência elétrica requerida para o bombeamento da água não disponibilizada à cultura, $\mathrm{kW}$

$\mathrm{H}_{\mathrm{m}}$ - altura manométrica do sistema, mca

$\mathrm{EA}_{80}$ - eficiência de aplicação para $80 \%$ da área adequadamente irrigada, $\%$

$\mathrm{P}_{\mathrm{EdPA}}$ - potência elétrica requerida para o funcionamento do emissor, $\mathrm{kW}$

$\mathrm{h}_{\mathrm{ASP}}$ - pressão nominal do aspersor, mca

$\mathrm{P}_{\text {Edasp }}$ - potência elétrica requerida na aspersão, $\mathrm{kW}$

Para avaliação da perda de energia no sistema de irrigação completo considerando a contribuição de cada elemento do pivô central, obteveram-se a Eq. 7 a partir da composição da potência elétrica dissipada no conjunto motobomba, a Eq. 5, com as potências dissipadas nas tubulações de sucção, adução e linha lateral, Eq. 6 e a potência requerida na aspersão, Eq. 4.

$$
\begin{gathered}
\mathrm{P}_{\mathrm{Edmb}}=\frac{\gamma \cdot \mathrm{EA}_{80} \cdot \mathrm{Q}_{\mathrm{S}} \cdot \mathrm{h}_{\mathrm{g}}}{100 \cdot \eta_{\mathrm{mb}}} \cdot\left(1-\eta_{\mathrm{mb}}\right) \\
\mathrm{P}_{\mathrm{Edt}}=\frac{\gamma \cdot \mathrm{EA}_{80} \cdot \mathrm{Q}_{\mathrm{S}}}{100 \cdot \eta_{\mathrm{mb}}} \cdot\left(\Delta \mathrm{h}_{\mathrm{s}}+\Delta \mathrm{h}_{\mathrm{Ad}}+\Delta \mathrm{h}_{\mathrm{LL}}\right) \\
\mathrm{P}_{\mathrm{Edi}}=\frac{\gamma \cdot \mathrm{EA}_{80} \cdot \mathrm{Q}_{\mathrm{S}}}{100 \cdot \eta_{\mathrm{mb}}} \cdot\left(\left(\mathrm{h}_{\mathrm{g}} \cdot\left(1-\eta_{\mathrm{mb}}\right)\right)+\right. \\
\left.\left(\Delta \mathrm{h}_{\mathrm{s}}+\Delta \mathrm{h}_{\mathrm{Ad}}+\Delta \mathrm{h}_{\mathrm{LL}}\right)+\left(\mathrm{H}_{\mathrm{m}} \cdot\left(\frac{100}{\mathrm{EA}_{80}}-1\right)+\mathrm{h}_{\mathrm{ASP}}\right)\right)
\end{gathered}
$$

em que:

\footnotetext{
$\mathrm{P}_{\mathrm{Edmb}}$ - potência elétrica dissipada no conjunto motobomba, $\mathrm{kW}$
}

$$
\begin{aligned}
\mathrm{hg} & \text { - desnível geométrico entre a captação e o emis- } \\
& \text { sor na condição mais desfavorável, } \mathrm{m} \\
\mathrm{P}_{\mathrm{Edt}} & \text { potência elétrica dissipada nas tubulações, } \mathrm{kW} \\
\Delta \mathrm{h}_{\mathrm{s}} & \text { - perda de carga na sucção, mca } \\
\Delta \mathrm{h}_{\mathrm{Ad}} & - \text { perda de carga na adutora, mca } \\
\Delta \mathrm{h}_{\mathrm{LL}} & \text { - perda de carga na linha lateral, mca } \\
\mathrm{P}_{\mathrm{Edi}} & \text { - potência elétrica dissipada no sistema de irri- } \\
& \text { gação, } \mathrm{kW}
\end{aligned}
$$

\section{Avaliação econômica}

A avaliação econômica dos pivôs determinou o benefício específico pela diferença entre o custo anual específico da energia dissipada para duas configurações de eficiência energética (Eq. 8, 9, 10, 11 e 12). A avaliação econômica foi realizada através do custo anual específico, relação benefício custo e tempo de retorno de capital, EMBRAPA (2005).

$$
\begin{gathered}
\mathrm{Q}_{80}=3,6 \cdot \mathrm{Q}_{\mathrm{S}} \cdot \mathrm{EA}_{80} \\
\mathrm{I}_{\mathrm{e}}=\frac{\mathrm{I}}{\mathrm{Q}_{80}} \\
\mathrm{E}_{\mathrm{de}}=\frac{\mathrm{P}_{\mathrm{Edi}}}{\mathrm{Q}_{80}} \\
\mathrm{C}_{\mathrm{Ede}}=\mathrm{E}_{\mathrm{de}} \cdot \mathrm{t} \cdot \mathrm{C}_{\mathrm{ME}} \\
\mathrm{B}_{\mathrm{e}}=\mathrm{C}_{\mathrm{Ede}(\mathrm{i})}-\mathrm{C}_{\mathrm{Ede}(\mathrm{i}+1)}
\end{gathered}
$$

em que:

$\mathrm{Q}_{80}$ - vazão útil considerando-se eficiência de aplicação para $80 \%$ da área adequadamente irrigada, $\mathrm{mm}$ ha $\mathrm{h}^{-1}$

$I_{e}$ - investimento específico para uma medida de eficiência energética, $R \$ \mathrm{~mm}^{-1} \mathrm{ha}^{-1}$

I - investimento de uma medida de eficiência energética, $\mathrm{R} \$$

$\mathrm{E}_{\mathrm{de}}$ - energia dissipada específica no sistema de irrigação, $\mathrm{kWh} \mathrm{mm} \mathrm{mm}^{-1} \mathrm{ha}^{-1}$

$\mathrm{C}_{\mathrm{Ede}}$ - Custo anual específico da energia dissipada, $\mathrm{R} \$$ $\mathrm{mm}^{-1} \mathrm{ha}^{-1} \mathrm{ano}^{-1}$

$\mathrm{t}$ - horas de funcionamento anual, $\mathrm{h}$

$\mathrm{B}_{\mathrm{e}}$ - benefício anual específico, $\mathrm{R} \$ \mathrm{~mm}^{-1} \mathrm{ha}^{-1}$

$\mathrm{C}_{\mathrm{ME}}$ - custo médio da energia elétrica, $\mathrm{R} \$ \mathrm{kWh}^{-1}$

O custo médio da energia foi determinado para 1260 horas de funcionamento no horário fora de ponta seco e 140 h no úmido. Para o horário reservado foram utilizadas $600 \mathrm{~h}$, das quais 540 horas no período seco e 60 horas no úmido (Frizzone \& Dourado Neto, 2003), considerando-se os procedimentos e as tarifas em vigor, ANEEL (2000; 2006).

\section{Caracterização energética de um pivô central}

A caracterização completa do pivô foi definida pelo consumo específico normalizado do sistema de irrigação (Eq. 13) e pela eficiência energética na irrigação proposta (Eq. 14), determinada pela relação entre a potência útil na irrigação (Eq. 15) e a potência elétrica no conjunto motobomba. 


$$
\begin{gathered}
\mathrm{C}_{\mathrm{ENI}}=\gamma \cdot \frac{\mathrm{H}_{\mathrm{m}}}{\mathrm{h}_{\mathrm{g}} \cdot \mathrm{EA}_{80} \cdot \eta_{\mathrm{mb}}} \cdot \frac{10^{4}}{360} \\
\mathrm{E}_{\mathrm{I}}=\mathrm{EA}_{80} \cdot \frac{\mathrm{h}_{\mathrm{g}}}{\mathrm{H}_{\mathrm{m}}} \cdot \eta_{\mathrm{mb}} \\
\mathrm{P}_{\mathrm{UI}}=\gamma \cdot \mathrm{h}_{\mathrm{g}} \cdot \frac{\mathrm{Q}_{\mathrm{S}} \cdot \mathrm{EA}_{80}}{100}
\end{gathered}
$$

em que:

$\mathrm{C}_{\mathrm{ENI}}$ - consumo de energia normalizado na irrigação em $100 \mathrm{~m}$ de altura geométrica, kWh $\mathrm{mm}^{-1} \mathrm{ha}^{-1} 10^{-2} \mathrm{~m}^{-1}$

$E_{I}$ - eficiência energética na irrigação, \%

$\mathrm{P}_{\mathrm{UI}}$ - potência útil na irrigação, $\mathrm{kW}$

$\mathrm{O}$ consumo específico normalizado proposto determina a energia necessária para irrigar um milímetro em pelo menos $80 \%$ de um hectare, quando a altura geométrica é igual a $100 \mathrm{~m}$.

\section{RESULTADOS E DISCUSSÃO}

Os resultados obtidos na avaliação hídrica dos equipamentos são apresentados na Tabela 2. Vazamentos na linha late- ral, perdas por evaporação e deriva pelo vento e, mais significativamente, o grande volume de água percolada abaixo da zona radicular das culturas, proporcionaram as baixas eficiências de aplicação de água.

Apesar do desgaste e do uso de aspersores fora das especificações, os coeficientes de uniformidade de Heerman e Hein foram bons, acima de 85\%, para os pivôs L06 e G13, mas, a uniformidade foi baixa para o pivô C08, conforme ABNT (1998).

As perdas de carga e o rendimento dos conjuntos motobombas são apresentados na Tabela 3 . O funcionamento fora do ponto de rendimento máximo e os desgastes das bombas resultaram nos baixos rendimentos obtidos nos pivôs G13 (59\%) e C08 (63\%); já as elevadas velocidades observadas de até $5 \mathrm{~m} \mathrm{~s}^{-1}$, o material e o envelhecimento da tubulação, justificam as perdas de carga encontradas nas adutoras e linhas laterais.

O fluxo energético dos pivôs avaliados, Tabela 4, e os custos anuais específicos da energia dissipada, são apresentados na Figura 1, considerando-se o custo médio da energia igual a $0,177485 \mathrm{R} \$ \mathrm{kWh}^{-1}$.

Avaliando-se a implementação de medidas de eficiência energética apresentam-se, na Tabela 5, as características ob-

\begin{tabular}{|c|c|c|c|c|c|c|c|c|c|}
\hline \multirow{2}{*}{\multicolumn{2}{|c|}{ Pivô }} & \multirow{2}{*}{ Vazão $\left(m^{3} s^{-1}\right)$} & \multirow{2}{*}{$\begin{array}{l}\text { Tempo de uma } \\
\text { volta (h) }\end{array}$} & \multirow{2}{*}{$\begin{array}{l}\text { Lâmina } \\
\text { bruta (mm) }\end{array}$} & \multirow{2}{*}{$\mathrm{C}_{\mathrm{uH}} *(\%)$} & \multirow{2}{*}{$\begin{array}{l}\text { Eficiência de } \\
\text { aplicação** } \\
\text { (\%) }\end{array}$} & \multicolumn{3}{|c|}{ Condições Climáticas médias } \\
\hline & & & & & & & Temperatura (C) & U. $\mathbf{R}^{* * *}(\%)$ & Vento $\left(\mathrm{m} \mathrm{s}^{-1}\right)$ \\
\hline 1 & L06 & 0,130 & 56,0 & 22,2 & 89,2 & 78,8 & 30,4 & 64 & 3,9 \\
\hline 2 & $\mathrm{C08}$ & 0,077 & 112,0 & 27,2 & 72,5 & 59,1 & 30,5 & 40 & 1,6 \\
\hline 3 & G13 & 0,104 & 76,1 & 26,4 & 86,7 & 78,6 & 27,0 & 51 & 0,9 \\
\hline
\end{tabular}
tidas quando se simula a substituição do motor, da bomba,

Tabela 2. Avaliação dos pivôs

\begin{tabular}{|c|c|c|c|c|c|c|c|c|c|c|}
\hline \multirow{2}{*}{\multicolumn{2}{|c|}{ Pivô }} & \multicolumn{4}{|c|}{ Perda de carga (mca) } & \multirow{2}{*}{$\begin{array}{c}\text { Desnível } \\
\text { geométrico } \\
\text { (m) }\end{array}$} & \multirow{2}{*}{$\begin{array}{c}\text { Altura } \\
\text { manométrica } \\
\text { (mca) }\end{array}$} & \multirow{2}{*}{$\begin{array}{l}\text { Potência } \\
\text { hidráulica } \\
\text { (kW) }\end{array}$} & \multirow{2}{*}{$\begin{array}{l}\text { Potência } \\
\text { elétrica } \\
\text { (kW) }\end{array}$} & \multirow{2}{*}{$\begin{array}{l}\text { Rendimento da } \\
\text { motobomba } \\
\text { (adimensional) }\end{array}$} \\
\hline & & sucção & adutora & linha lateral & aspersão & & & & & \\
\hline 1 & L06 & 0,7 & 23,2 & 41,4 & 14,1 & 36,0 & 115,3 & 147,0 & 197,0 & 0,75 \\
\hline 2 & $\mathrm{C} 08$ & 0,4 & 85,3 & 29,8 & 14,1 & 39,7 & 169,2 & 128,2 & 203,1 & 0,63 \\
\hline 3 & G13 & 0,8 & 12,1 & 51,2 & 14,1 & 64,4 & 142,5 & 145,6 & 247,6 & 0,59 \\
\hline
\end{tabular}

* Coeficiente de uniformidade de Heerman e Hein; ** Para $80 \%$ da área adequadamente irrigada; *** Umidade relativa do ar

Tabela 3. Rendimento dos conjuntos motobombas

\begin{tabular}{|c|c|c|c|c|c|c|c|c|c|c|}
\hline \multirow{2}{*}{\multicolumn{2}{|c|}{ Pivô }} & \multirow{3}{*}{$\begin{array}{c}\mathbf{Q}_{80} \\
\left(\mathrm{~mm} \mathrm{ha} \mathrm{h}^{-1}\right) \\
36,70\end{array}$} & \multicolumn{7}{|c|}{ Energia específica $\left(\mathrm{kWh} \mathrm{mm}^{-1} \mathrm{ha}^{-1}\right)$} & \multirow{3}{*}{ 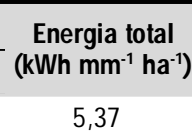 } \\
\hline & & & \multirow{2}{*}{$\begin{array}{c}\text { Motobomba } \\
1,36\end{array}$} & \multirow{2}{*}{$\begin{array}{c}\text { Sucção } \\
0,03\end{array}$} & \multirow{2}{*}{$\begin{array}{c}\text { Adutora } \\
1,08\end{array}$} & \multirow{2}{*}{$\begin{array}{c}\text { Linha lateral } \\
1,93\end{array}$} & \multirow{2}{*}{$\begin{array}{c}\text { Aspersão } \\
1,67\end{array}$} & \multirow{2}{*}{$\begin{array}{l}\text { Útil } \\
0,98\end{array}$} & \multirow{2}{*}{\begin{tabular}{|l|} 
Total \\
4,39 \\
\end{tabular}} & \\
\hline 1 & L06 & & & & & & & & & \\
\hline 2 & $\mathrm{C} 08$ & 16,37 & 4,57 & 0,03 & 6,25 & 2,18 & 5,70 & 1,08 & 11,32 & 12,40 \\
\hline 3 & $\mathrm{G} 13$ & 29,82 & 3,42 & 0,04 & 0,70 & 2,98 & 2,35 & 1,75 & 6,54 & 8,30 \\
\hline
\end{tabular}

Tabela 4. Energia específica requerida nos elementos dos sistemas de irrigação

\begin{tabular}{|c|c|c|c|c|c|c|c|c|c|c|c|}
\hline & & \multicolumn{10}{|c|}{ Variação das características para as medidas de eficiência energética analisadas } \\
\hline \multirow{2}{*}{\multicolumn{2}{|c|}{ Pivô }} & \multicolumn{4}{|c|}{ Rendimento (adimensional) } & \multicolumn{4}{|c|}{ Perda de carga (mca) } & \multirow{2}{*}{\multicolumn{2}{|c|}{$\begin{array}{c}\text { Eficiência de aplicação (\%) } \\
\text { Aspersores e reguladores de } \\
\text { pressão }\end{array}$}} \\
\hline & & \multicolumn{2}{|c|}{ Motor } & \multicolumn{2}{|c|}{ Bomba } & \multicolumn{2}{|c|}{ Adutora } & \multicolumn{2}{|c|}{ Linha lateral } & & \\
\hline 1 & L06 & 0,945 & 0,955 & 0,790 & 0,800 & 23,2 & 5,1 & 41,4 & 29,5 & 78,4 & 90,0 \\
\hline 2 & $\mathrm{C08}$ & 0,949 & 0,955 & 0,665 & 0,800 & 85,3 & 12,6 & 29,8 & 19,8 & 58,9 & 90,0 \\
\hline 3 & G13 & 0,950 & 0,955 & 0,619 & 0,800 & 12,1 & 12,1 & 51,2 & 34,7 & 79,5 & 90,0 \\
\hline
\end{tabular}

Tabela 5. Características das medidas de eficiência energética simuladas para os pivôs 


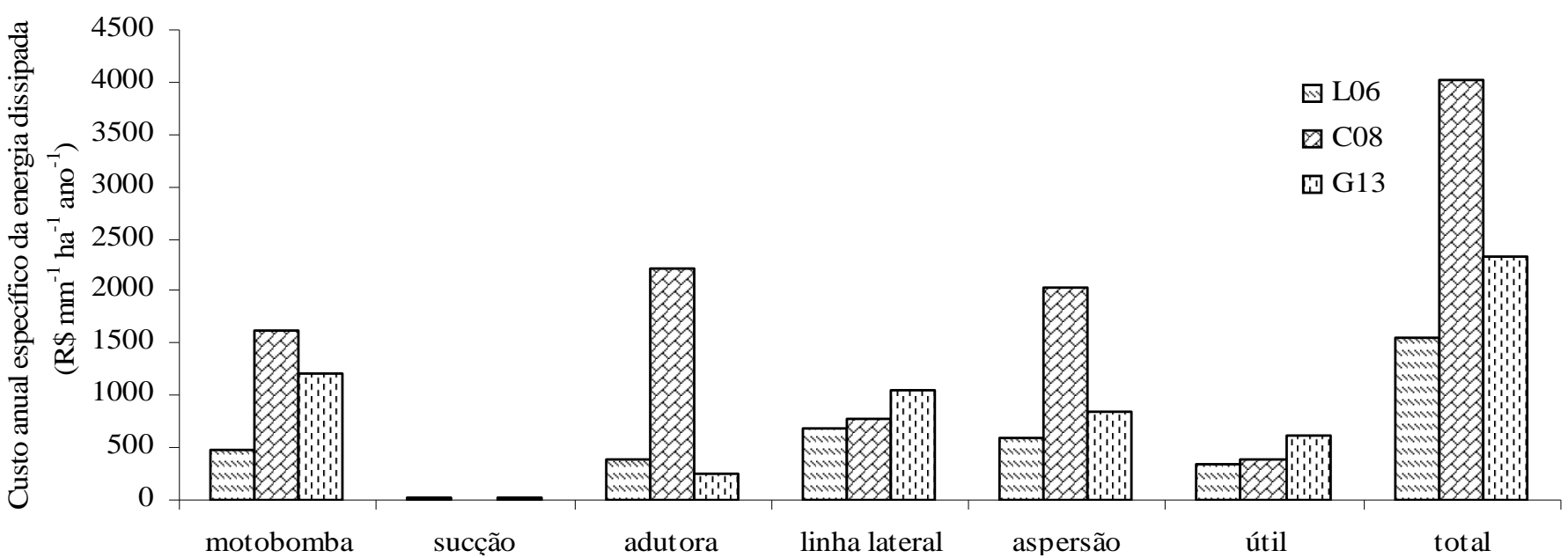

Figura 1. Custo anual específico da energia dissipada nos pivôs

da adutora por outra em tubo de PVC com diâmetro imediatamente superior, de um trecho de $156 \mathrm{~m}$ da tubulação aérea de diâmetro igual 168 por $203 \mathrm{~mm}$ e dos aspersores e reguladores de pressão.

As medidas de eficiência energética analisadas trazem como benefícios anuais, a redução dos gastos com a energia elétrica dissipada, conforme apresentado na Tabela 6; não se avaliou a substituição da adutora do pivô G13, já que ela se apresentava com perda de carga reduzida.

Tabela 6. Benefícios anuais específicos das medidas de eficiência energética avaliadas para os pivôs ensaiados no Assentamento Itamarati I, em abril de 2006

\begin{tabular}{|c|c|c|c|c|c|c|}
\hline \multirow{2}{*}{\multicolumn{2}{|c|}{ Pivô }} & \multicolumn{5}{|c|}{$\begin{array}{l}\text { Benefício anual específico das medidas de } \\
\text { eficiência energética } \quad\left(R \$ \mathrm{~mm}^{-1} \mathrm{ha}^{-1}\right)\end{array}$} \\
\hline & & Moto & Bomba & Adutora & $\begin{array}{l}\text { Linha } \\
\text { lateral }\end{array}$ & Aspersão \\
\hline 1 & L06 & 19,59 & 24,08 & 298,63 & 196,74 & 244,63 \\
\hline 2 & $\mathrm{C} 08$ & 26,47 & 764,77 & $1.892,35$ & 257,85 & $1.520,69$ \\
\hline 3 & $\mathrm{G} 13$ & 14,69 & 666,30 & & 342,57 & 342,27 \\
\hline
\end{tabular}

A partir dos investimentos necessários para a implementação das medidas de eficiência energética, Tabela 7, se determinaram os investimentos específicos e seus custos anuais, para uma taxa de oportunidade igual a $8,8 \%$ ao ano e horizonte de análise de 15 anos.

Sugere-se, com base na avaliação econômica, que primeiro se substituam os aspersores e reguladores de pressão seguidos das bombas dos pivôs $\mathrm{C} 08$ e G13 e a adutora do pivô C08, de acordo com a Tabela 8 .
Tabela 8. Análise econômica dos investimentos para implementação de medidas de eficiência energética dos pivôs ensaiados no Assentamento Itamarati I, em abril de 2006

\begin{tabular}{|c|c|c|c|c|c|c|c|}
\hline \multirow{3}{*}{\multicolumn{2}{|c|}{ Pivô }} & \multirow{2}{*}{\multicolumn{2}{|c|}{$\begin{array}{c}\text { Primeira avaliação } \\
\text { Asperssores }\end{array}$}} & \multirow{2}{*}{\multicolumn{2}{|c|}{$\frac{\text { Segunda avaliação }}{\text { Bomba }}$}} & \multirow{2}{*}{\multicolumn{2}{|c|}{$\begin{array}{c}\text { Terceira avaliação } \\
\text { Adutora }\end{array}$}} \\
\hline & & & & & & & \\
\hline & & $B / C(1)$ & TRC (anos) & $B / C(1)$ & TRC (anos) & $B / C(1)$ & TRC (anos) \\
\hline 1 & L06 & 4,23 & 2,21 & 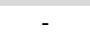 & 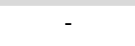 & 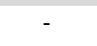 & - \\
\hline 2 & $\mathrm{C} 08$ & 23,97 & 0,36 & 3,65 & 2,60 & 2,00 & 5,26 \\
\hline 3 & G13 & 7,56 & 1,18 & 6,16 & 1,47 & - & - \\
\hline
\end{tabular}

* Relação benefício custo; ** Tempo de retorno de capital

Realizada a análise econômica, o sistema pode ser caracterizado quanto ao uso da energia; desta forma, a eficiência energética na irrigação e o consumo específico normalizado na irrigação, são apresentados nas Figuras 2A e B.

De acordo com a Figura 2A, pode-se observar que a eficiência energética na irrigação atual varia de 8,7 e 21,1\% nos pivôs C08 e G13; esta eficiência, por outro lado, determina, para os sistemas ensaiados, que até $91,3 \%$ da energia absorvida pelo motor são dissipadas no conjunto motobomba em virtude do seu rendimento, nas tubulações de suç̧ão, adução e da linha lateral por atrito, nos reguladores de pressão e nos emissores por evaporação, deriva pelo vento, vazamentos, percolação profunda e para formação e distribuição das gotas.

O consumo de energia normalizado na irrigação, Figura 2B, determina que, com 12,89 kWh, o pivô G13 fornece um milímetro de água em pelo menos $80 \%$ da área irrigada quando a altura geométrica é igual a $100 \mathrm{~m}$, enquanto os pivôs L06 e C08 necessitam de 14,91 e $31,24 \mathrm{kWh} \mathrm{mm}^{-1} \mathrm{ha}^{-1} 10^{-2}$ $\mathrm{m}^{-1}$, respectivamente.

Tabela 7. Investimento para implementação de medidas de eficiência energética dos pivôs

\begin{tabular}{|c|c|c|c|c|c|c|c|c|c|c|c|}
\hline \multirow{2}{*}{\multicolumn{2}{|c|}{ Pivô }} & \multicolumn{10}{|c|}{ Investimento (R\$) e custo anual específico (R\$ $\left.\mathrm{mm}^{-1} \mathrm{ha}^{-1}\right)$} \\
\hline & & \multicolumn{2}{|c|}{ Motor } & \multicolumn{2}{|c|}{ Bomba } & \multicolumn{2}{|c|}{ Adutora } & \multicolumn{2}{|c|}{ Linha lateral } & \multicolumn{2}{|c|}{ Aspersão } \\
\hline 1 & L06 & 44.000 & 146,98 & 28.000 & 93,54 & 281.000 & 938,69 & 124.000 & 414,23 & 19.872 & 57,84 \\
\hline 2 & $\mathrm{C} 08$ & 52.000 & 389,34 & 28.000 & 209,65 & 104.200 & 780,19 & 124.000 & 928,44 & 12.946 & 63,44 \\
\hline 3 & G13 & 52.000 & 213,77 & 26.300 & 108,12 & - & - & 124.000 & 509,76 & 12.461 & 45,26 \\
\hline
\end{tabular}




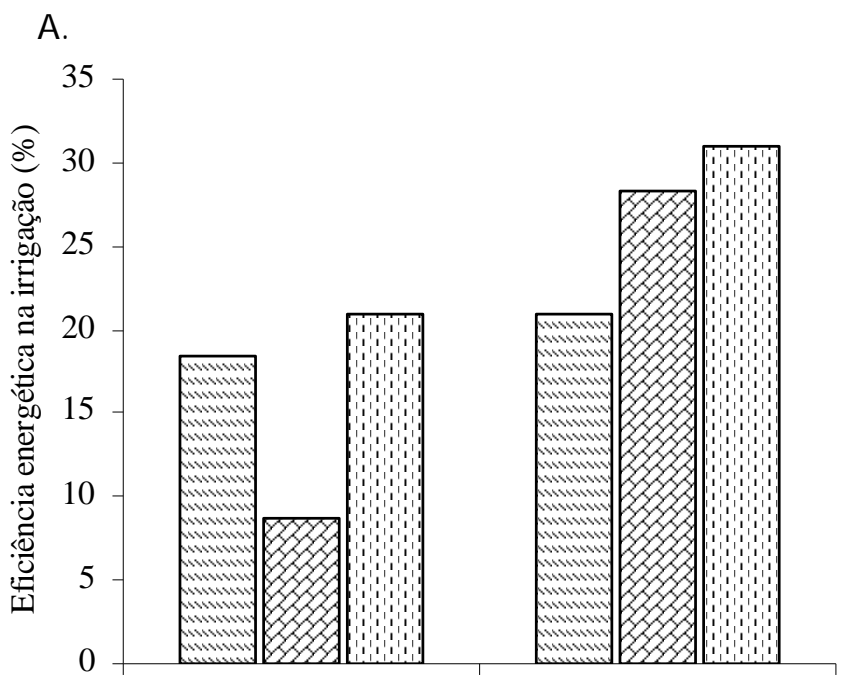

B.

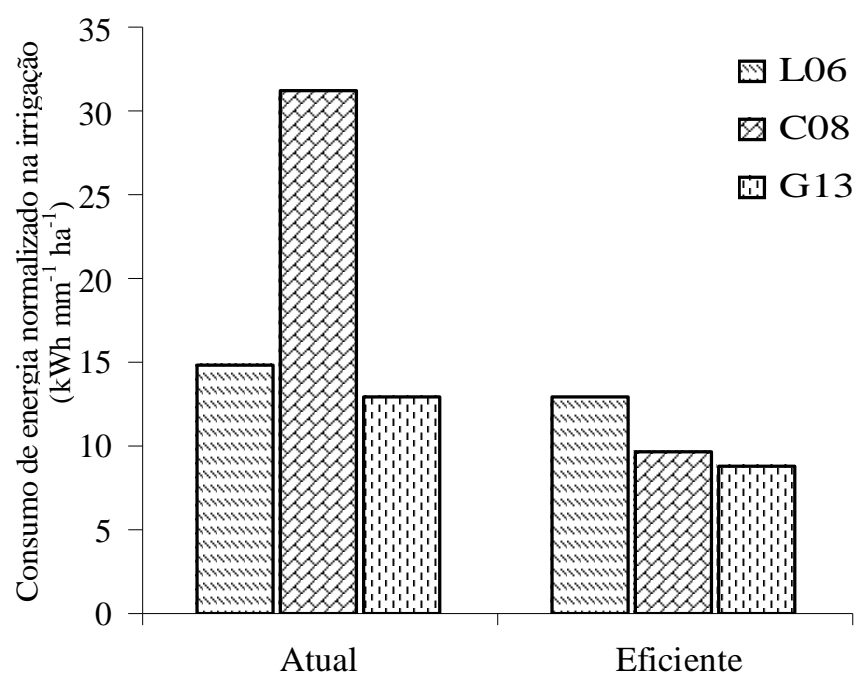

Figura 2. Caracterização energética dos pivôs

Extrapolando os valores médios das eficiências avaliadas aos demais equipamentos do Assentamento Itamarati I, é possível se obter uma economia de 118,8 mil reais anuais nas faturas de energia (Tabela 9).

Tabela 9. Extrapolação da economia média obtida das medidas de eficiência energética simuladas para os sistema de irrigação

\begin{tabular}{|c|c|c|c|c|}
\hline \multirow{2}{*}{ Configuração } & \multirow{2}{*}{$\begin{array}{c}\text { Eficiência } \\
\text { energética } \\
\text { média de } \\
\text { imigação }(\%)\end{array}$} & \multirow{2}{*}{$\begin{array}{c}\text { Custo da } \\
\text { energia } \\
\text { dissipada (R\$) }\end{array}$} & \multicolumn{2}{|c|}{ Economia } \\
\hline & & & (R\$) & (\%) \\
\hline Atual & 14,9 & $918.087,55$ & \multirow{2}{*}{$118.772,78$} & \multirow{2}{*}{11,0} \\
\hline Simulada & 25,9 & 799.314,77 & & \\
\hline
\end{tabular}

\section{CONCLUSÕES}

1. Os projetos de pivôs centrais de irrigação devem contemplar a análise econômica de investimento, não apenas da adutora, mas, também, do motor, da bomba, da linha lateral e dos aspersores.
2. A avaliação periódica e sistemática de um sistema de irrigação instalado pode fornecer o momento correto para a intervenção no equipamento, considerando-se as características reais de utilização e custo médio de energia.

3. Os sistemas de irrigação do Assentamento Itamarati I avaliados, apresentaram perda de energia de até $91,3 \%$ devido, principalmente, aos desgastes e ao uso incorreto dos aspersores, perda de carga na adutora e linha lateral e baixo rendimento do conjunto motobomba.

4. Com as medidas de eficiência energética simuladas foi possível se obter rendimento médio de $25,9 \%$, com redução média anual dos gastos com energia elétrica de 118,8 mil reais, em todo o sistema de irrigação do Assentamento Itamarati I.

5. Propõe-se, então, a complementação e padronização das informações obtidas do coeficiente de uniformidade Heerman e Hein e da eficiência de aplicação, normalmente utilizados para avaliar pivôs de irrigação com os propostos consumo específico normalizado e eficiência energética na irrigação.

6. Os indicadores de eficiência propostos trazem informações importantes e necessárias para avaliação durante a compra e utilização desses equipamentos e, sobretudo, podem propiciar a concorrência por melhores desempenhos energéticos entre fabricantes, projetistas e fornecedores.

\section{LITERATURA CITADA}

ABNT - Associação Brasileira de Normas Técnicas. NBR 6397 - Ensaios em bombas hidráulicas de fluxo. Rio de Janeiro: ABNT, 1975. 48p.

ABNT - Associação Brasileira de Normas Técnicas. NBR 14244 Equipamento de irrigação mecanizada: Pivô central e lateral móvel providos de emissores fixos ou rotativos, determinação da uniformidade de distribuição de água. Rio de Janeiro: ABNT, 1998. 11p.

ANEEL - Agência Nacional de Energia Elétrica. Resolução n. 456 de 29.nov.00. Condições de fornecimento de energia elétrica, 2000. 80p. http://www.aneel.gov.br/. 10. Set. 2006.

ANEEL - Agência Nacional de Energia Elétrica. Resolução homologatória n. 311, de 6.abril.06. Homologa as tarifas de fornecimento de energia elétrica da empresa energética de Mato Grosso do Sul S/A - ENERSUL para o período de 08 de abril de 2006 a 07 de abril de 2007. http://www.aneel.gov.br/. 10 Set. 2006.

Batista, M. B.; Coelho, M. M. L. P. Fundamentos de engenharia hidráulica. Belo Horizonte: UFMG 2003. 440p.

Bernardo, S.; Soares, A. A.; Mantovani, E. C. Manual de irrigação. 7.ed. Viçosa: UFV, 2005. 611p.

CLASP - Collaborative Labeling and Appliance Standards Program. Energy-Efficiency Labels and Standards: A guidebook for appliances, equipment, and lighting. Washington. 2.ed., 2005. 321p. http://www.clasponline.org/download/ General/2001/211/ index.php3. 21 Jun 2005.

EMBRAPA - Empresa Brasileira de Pesquisas Agropecuária. Planejamento de irrigação: Análise de decisão de investimento. Andrade Jr., A.S; Moretti, J. L; Zocoler, J. L. (ed.) Brasília: Embrapa Informação Tecnológica, 2005. 626p. 
Frizzone, J. A.; Dourado Neto, A. D. Avaliação de sistema de irrigação. In: Miranda, J. H.de; Pires, R. C. de M. Irrigação: Engenharia agrícola, irrigação. Piracicaba: SBEA, FUNEP, v.2, 2003, p.573-651.

Gomes, H. P. Sistema de abastecimento de água: Dimensionamento econômico e operação de redes e elevatória. 2.ed. João Pessoa: UFPB, 2004. 242p.
Oliveira, A. S.; Pereira, F. A. C.; Paz, V. P. S.; Santos, C. A. Avaliação do desempenho de sistemas pivô central na região oeste da Bahia. Irriga, v.9, n.2, p.126-135, 2004.

Zocoler, J. L. Análise econômica de sistema de irrigação. In: Miranda, J. H.de; Pires, R. C. de M. Irrigação: Engenharia agrícola irrigação. Piracicaba: SBEA, FUNEP, v.2, 2003, p.653-703. 